\title{
Sleep Stage, Sleep Duration, Attention Span
}

\author{
Hanfei $\mathrm{Cao}^{1, \mathrm{a}}$
}

\author{
${ }^{1}$ Komagome senior high school, Tokyo, Japan 1130022 \\ ${ }^{a}$ caohanfei21@gmail.com
}

\begin{abstract}
Have you ever woken up refreshed after only 4 hours sleep, and woken up listless after 8 hours sleep? Many works of literature reviewed how sleep deprivation affects people in many ways. While there has been much research on the problem of sleep deprivation, few researchers have considered sleep stage duration. In this study, we focus on the sleep stage instead, to find out whether a specific sleep duration affects people's attention span.
\end{abstract}

Keywords: NREM sleep, sleep stage, attention span

\section{INTRODUCTION}

Half a century ago, studies first demonstrated that people have rapid eye movement (REM) stage and nonrapid eye movement (NREM) stage during sleep states [1]. In the REM stage, people's heart rate and blood pressure increase, and breathing becomes faster [2]. In the NREM stage, including wakefulness to sleep, light sleep, and deep sleep, people can conserve brain energy and facilitate memory consolidation. Nonetheless, since both REM and NREM are distributed differently in one-time sleep, and these varied distrition can be consistent with mental disorders such as depressiom [6]. Therefore, the understanding of how these sleep durations directly relate to our attention span could contribute to future investigation on attention improvement

People spend more of the sleep cycles in light sleep than in other sleep stages. Also, the amount of NREM sleep is demonstrated to be positively related to the enhancement of cortical plasticity [3], and studies show that neural plasticity is related to several aspects of attention [4]. Thus, we hypothesized that people with a longer light sleep duration tend to have a longer attention span.

\section{METHODS}

\subsection{Participants}

Fifty individuals $(28 \mathrm{M}, 22 \mathrm{~F}$; age: $=28 \pm 11.8)$ participated in the study. There are 29 adolescents and 20 middle-aged participants. The age of adolescents ranges between 10 and 29 years old and the age of middle-aged participants ranges between 30 and 49 years old. One attention task was administrated once within 4 hours they wake up. Convenient sampling was used in the study, indicating that participants are readily approachable to be a part of the sample. Researchers chose people they know in this case. We assign participants who have a larger ratio of light sleep in total sleep duration to the light group, and assign participants who have a larger ratio of deep plus rem sleep in total sleep duration to deep\&rem group (deeprem group). All participants must be the IOS users to make sure that they can meet the procedures. A procedure guideline is sent to each participant to make sure that they can follow the procedures step by step. Participants had to install a sleep staging monitoring application on their phones and make sure that it is processing while they are asleep. Then, fill out an attention survey within 4 hours they wake up.

\subsection{Instrument}

\subsubsection{Sleep staging monitor}

Two main instruments were used in the study. Sleep score, which is a sleep staging monitor, claims it can detect the duration of each sleep stage using sonar technology [5]. Participants had to install this application on their phones and make sure that it is processing while they are asleep. Sleep score requires the accessibility to microphone to detect body movements and sound waves. Users must wave their hands around the phone before they start tracking to see if there is a wave shown on the screen. Before the application starts to work, it requires the participant to fill out the daily log, to see if they had alcohol, drugs, stress, sleepiness, and happiness. Then, 
they put the phone within a safe range so that the application can catch the movements, usually at the head of the bed. A record, including sleep duration of light sleep, deep sleep, and rem sleep, wake time, total sleep duration, will be available in the next day. Lastly, participants must report the record to the researcher by sending the screenshot of the record page.

\subsubsection{Attention span test}

Only after their sleep has been successfully recorded can they do the attention span survey as they might be too hyperactive while sleeping, moving their bodies out of the detectable range and eventually interrupt the record. The attention survey tests people's attention span. Participants will see either arrow pointed to the left or an arrow pointing to the right on the screen. By pressing ' $A$ ' on the keyboard while the arrow points to the left and ' $\mathrm{L}$ ' on the keyboard while the arrow points to the right, participants have a 2-second responding period to respond. The arrows will be shown constantly. The longest trials, which means their best score, will be recorded continually when they correctly respond during a period without any mistake. Once participants made a mistake in terms of wrongly matching the A or $\mathrm{L}$ with the direction of the arrow, trials will be counted all over again. Finally, after responding for around 3 minutes, participants can stop the test by long pressing the space keyboard. Participants must report a screenshot of the result. The longest trials without any mistake and maximum time without any mistake were analyzed in the study. The analysis focused on 3 parts. First, to see the difference in attention test performance in terms of maximum time and longest trials in light group and deeprem group, we compared the mean test result. Second, to see if the test result in both group is normal, we conducted the Shapiro test. Lastly, to compare whether there is a difference in both maximum time and longest trials for 2 groups, we used the Mann-Whitney U test.

\section{RESULTS}

Maximum time without any mistake (Test 1) and longest trials without any mistake (Test 2) were analyzed in the study. Data range is shown in Figure 1 and Figure 2. In the light group, both data of the tests in the Shapiro test show a $\mathrm{p}$-value less than 0.05 (test $1: \mathrm{p}=0.0065$ : test 2: $\mathrm{p}=0.0042$ ), indicating that the data is normally distributed. Test 1 and test 2 in deeprem group, however, show the non-significant result, indicating the data is not normally distributed (test $1: \mathrm{p}=0.2818$; test $2: \mathrm{p}=0.2524$ ). The light group tended to have better performance in both tests shown in Figure 3 and Figure 4. However, no significant differences were found in Mann Whitney U test. Since the p-value in both test 1 and test 2 are larger than 0.05 (test $1: \mathrm{p}=0.9089$; test $2: \mathrm{p}=0.9419$ ) we failed to reject the null hypothesis. Thus, we do not have compelling evidence to say that there is a difference in either maximum time result and longest trials result between light group and deeprem group.

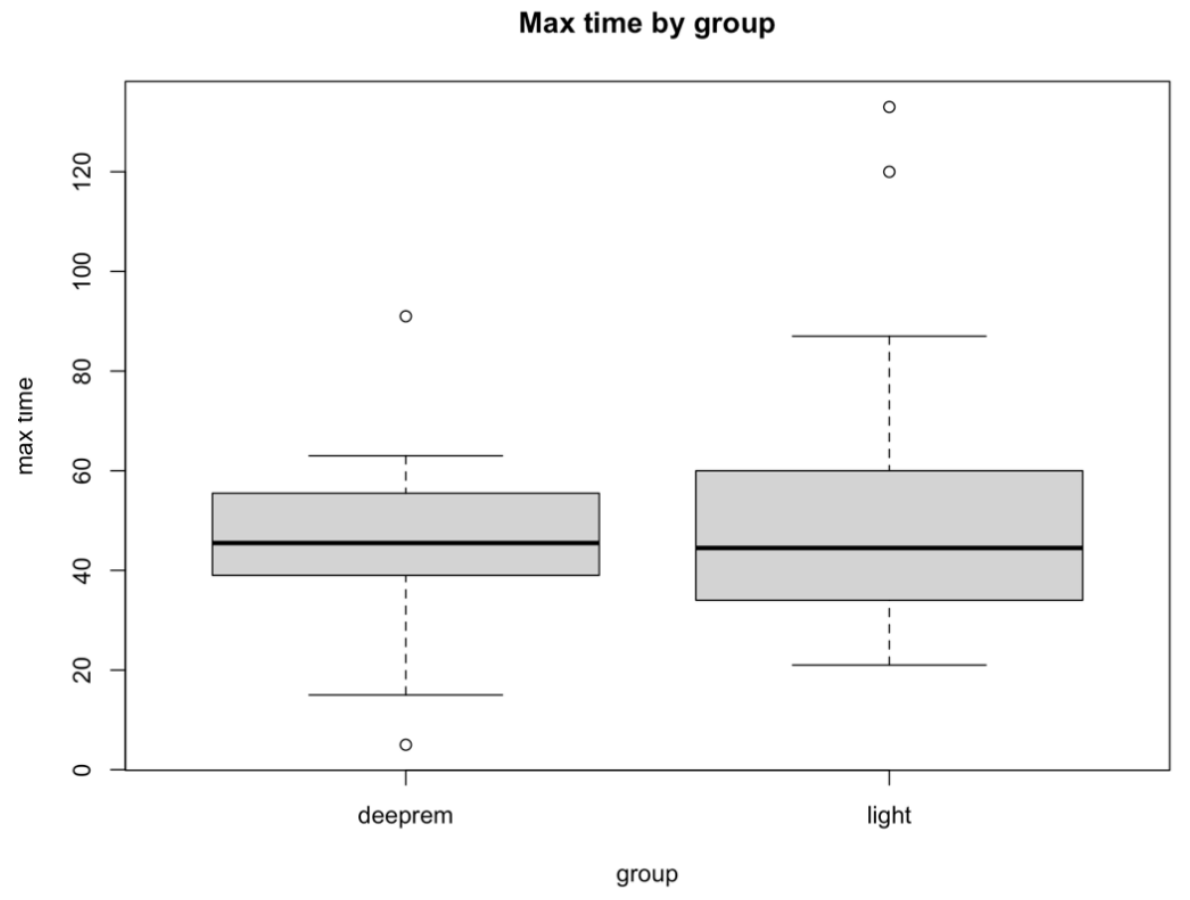

Figure 1 data of Test 1 in light group and deeprem group 


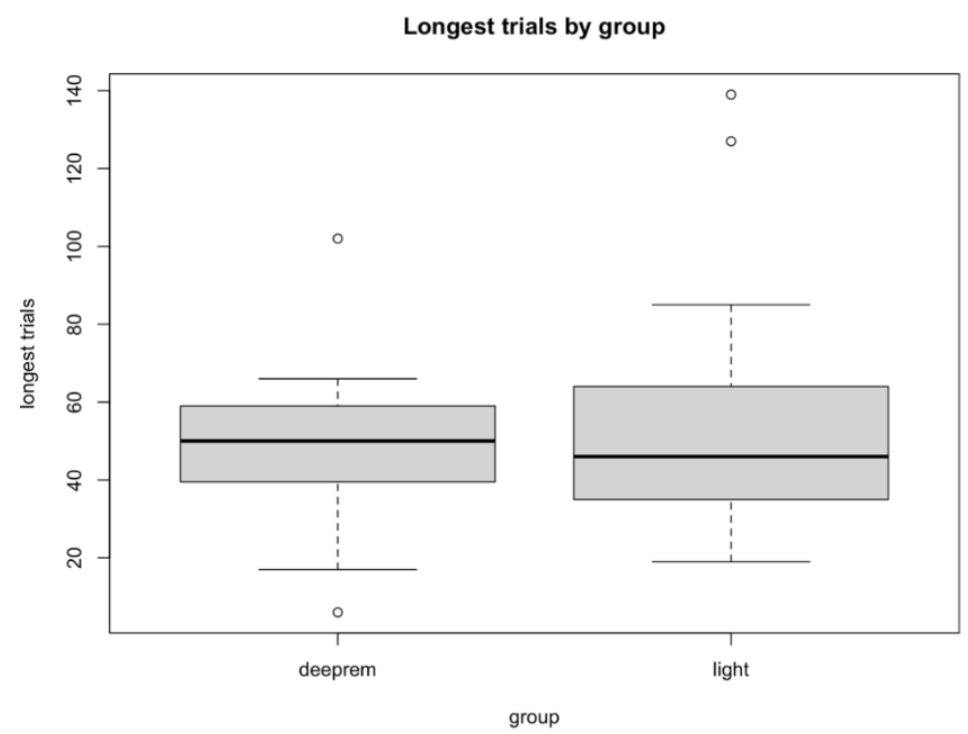

Figure 2 data of Test 2 in light group and deeprem group

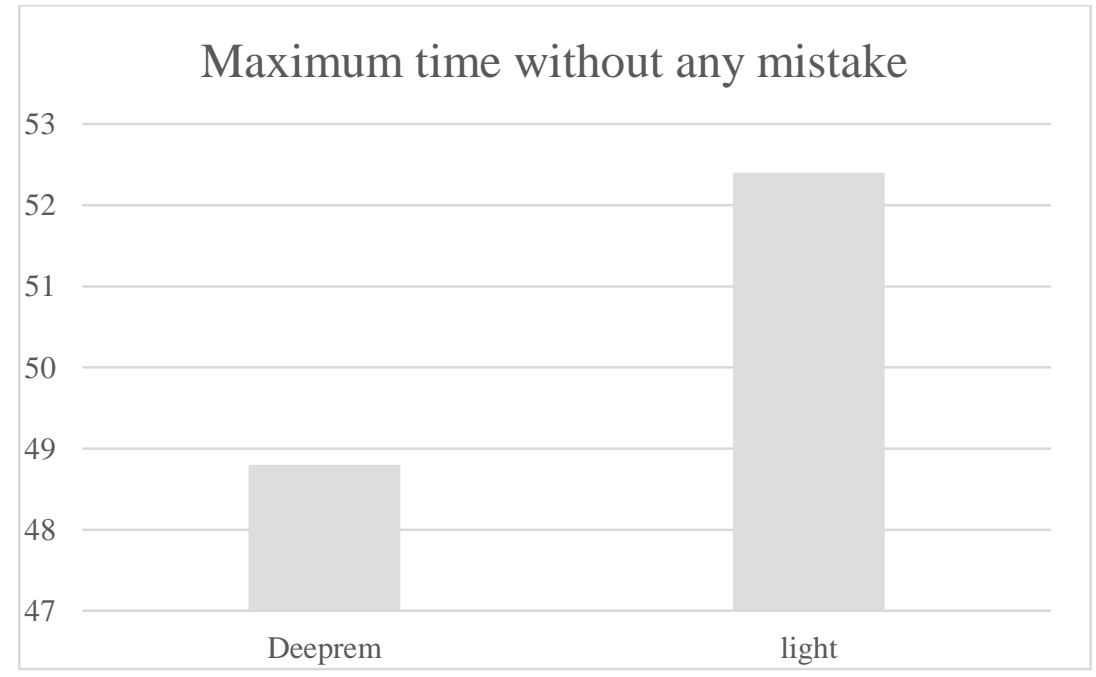

Figure 3 Test 1 in light group and deeprem group

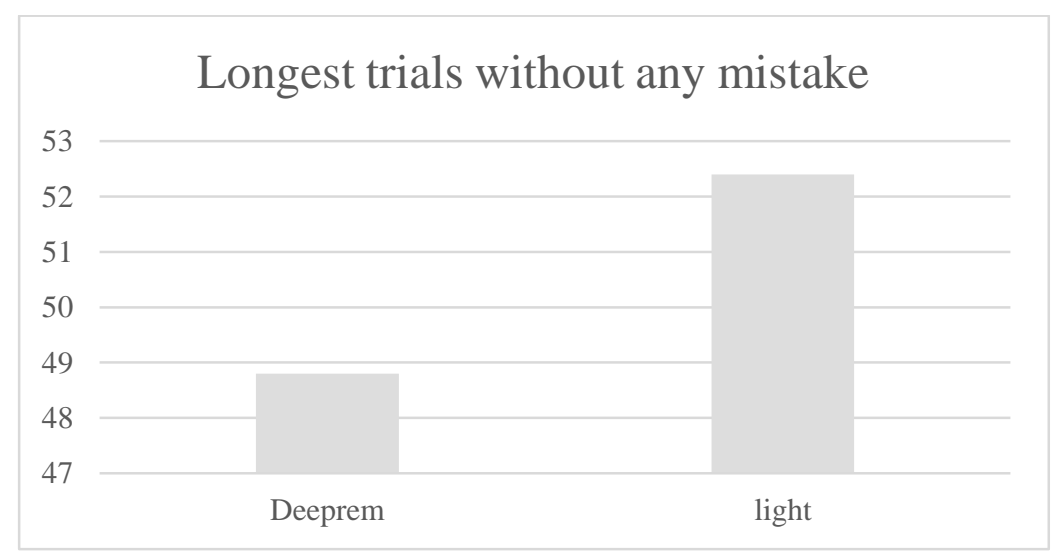

Figure 4 Test 2 in light group and deeprem group

\section{CONCLUSION}

In this study, we discussed the data from convenient samples, focusing on people's sleep duration in each sleep stage, and assigned people who have a bigger proportion of light sleep into light group and others into 
deeprem group. Additionally, by analyzing their 2 scores on attention span test respectively, we found only scores in light group are normally distributed. Finally, we couldn't support that there is a significant difference in 2 groups in neither test 1 nor test 2 . We failed to find evidence that can support our hypothesis that light sleep duration affect people's attention span. Although we failed to support our hypothesis, we ended up considering possible parameters that can be attributed to our result, which can be used to improve future studies on attention.

These results can be understood in the context of instruments. The attention test reported here is extremely easy to conduct but also limits the types of attention. Participants reported that they feel like the attention test has nothing to do with their sleep duration. In addition, by looking into the procedure, since we only required participants to do self-report, they may easily get irritated about responding to arrows and try to complete the attention test as soon as possible. Furthermore, by looking into the procedures, we find that there are many confounding variables. The cue is the daily log, we have no idea of participants' alcohol intake, drug intake, and stress level. It had a limitation in the self-report method. Another limitation was that its sample size was small.

In addition, only comparing light sleep duration, rem sleep duration, and deep sleep duration is less impossible since REM sleep duration only takes a small part in our total sleep duration. we arbitrarily divided groups into light and deeprem group.

What is the way forward? We hope that our review will embolden researchers to move beyond mere labeling of sleep duration toward a greater focus on other factors such as alcohol intake, drugs, and sleep deprivation. We need to know more about which factors contribute to our attention because they are functionally complementary.

\section{REFERENCES}

[1]. Aserinsky, E., \& Kleitman, N. (1953). Regularly occurring periods of eye motility, and concomitant phenomena, during sleep. Science, 118(3062), 273274.

[2]. Boudreau, P., Yeh, W. H., Dumont, G. A., \& Boivin, D. B. (2013). Circadian variation of heart rate variability across sleep stages. Sleep, 36(12), 19191928. https://doi.org/10.5665/sleep.3230

[3]. Frank, M. G., Issa, N. P., \& Stryker, M. P. (2001). Sleep enhances plasticity in the developing visual cortex. Neuron, 30(1), 275-287. https://doi.org/10.1016/s0896-6273(01)00279-3

[4]. McKay Moore Sohlberg. 2017. Attention Training. Encyclopedia of Clinical Neuropsychology, pages $1-2$. http://doi.org/10.1080/17588928.2017.1284775
[5]. Zaffaroni, A., Coffey, S., Dodd, S., Kilroy, H., Lyon, G., O'Rourke, D., Lederer, K., Fietze, I., \& Penzel, T. (2019). Sleep Staging Monitoring Based on Sonar Smartphone Technology. Annual International Conference of the IEEE Engineering in Medicine and Biology Society. IEEE Engineering in Medicine and Biology Society. Annual International Conference, 2019, 2230-2233. https://doi.org/10.1109/EMBC.2019.8857033

[6]. Wang, Y. Q., Li, R., Zhang, M. Q., Zhang, Z., Qu, W. M., \& Huang, Z. L. (2015). The Neurobiological Mechanisms and Treatments of REM Sleep Disturbances in Depression. Current neuropharmacology, 13(4), 543-553. https://doi.org/10.2174/1570159x13666150310002 540 